\title{
Assedi della guerra di Morea nel ciclo celebrativo di Francesco Morosini. Arte, topografia e storia militare
}

Sieges of the Morea War in the celebratory cycle of Francesco Morosini. Art, topography and military history

\author{
Elisabetta Molteni ${ }^{a}$, Alberto Pérez Negrete ${ }^{b}$ \\ a Università Ca' Foscari Venezia, Venice, Italy, elieni@unive.it \\ b Universidad de Sevilla, Seville, Spain / Istituto Universitario di Architettura di Venezia, Venice, Italy, \\ albertopereznegrete@gmail.com
}

\begin{abstract}
The forty-eight paintings executed between the seventeenth and eighteenth centuries to celebrate the military campaigns of Francesco Morosini (1619-1694) are an exceptional repertoire of military genre painting. The canvas uses different figurative registers to represent naval battles, cities and territories, siege operations. If the relations with war literature and propaganda prints, which spread across Europe and which had their official "historiographer" in Vincenzo Coronelli in Venice, are evident, equally strong relationships can be established between the paintings, war reports and the plans made on the battlefield by military engineers. This paper deals with the paintings dedicated to the sieges of Corone and Negroponte are examined here.
\end{abstract}

Keywords: Francesco Morosini, Morea, war, siege, history painting.

\section{Pitture di guerra. Le "battaglie" Morosini}

Fino al 1889, nel palazzo di famiglia del doge Francesco Morosini (1619-1694) in campo Santo Stefano a Venezia, si trovava una serie di 48 dipinti dedicata alle più importanti imprese di guerra marittima e agli assedi di piazzeforti e città guidati dal doge e capitano generale da mar. Tutti i dipinti, acquistati dal Comune di Venezia dagli ultimi eredi di questo ramo dei Morosini, passarono allora alle Raccolte Civiche (Tonini, 2013). Le tele, di grandi dimensioni, furono esposte al $\mathrm{Mu}-$ seo Correr fino alla riorganizzazione delle sale eseguita da Carlo Scarpa quando furono quasi tutte confinate nei depositi o cedute in comodato a vari istituti militari cittadini. Solo recentemente il ciclo delle "battaglie" Morosini è stato in buona parte ricomposto e presentato al pubblico e agli studiosi ${ }^{1}$ ma ancora manca un'indagine puntuale che stabilisca le circostanze in cui il ciclo fu ideato e quando furono eseguiti i dipinti -opere di vari pittori, probabilmente realizzati in momenti diversi (Pignatti, 1960, pp. 254-256; Arisi, 1982)-e che renda conto delle ragioni che portarono a vari rimaneggiamenti delle tele, modifiche in alcuni casi molto evidenti sia nelle dimensioni (ad esempio sono state ridotte le tele di Lepanto, Museo Correr, Cl. I, 1346 e Corinto, 1366) che nel contenuto dei dipinti. In ogni tela, una lunga didascalia sotto alla scena descrive i fatti rappresentati e anche questi testi appaiono in molti casi riscritti e in altri aggiunti in un momento successivo rispetto all'esecuzione del dipinto. Molto probabilmente questi interventi sono stati eseguiti per adattarli alle pareti del "portego" maggiore di palazzo Mo- 
rosini, dove si trovavano al momento dell'acquisto da parte del Comune, collocati all'interno di una più complessa scenografia celebrativa del doge-condottiero.

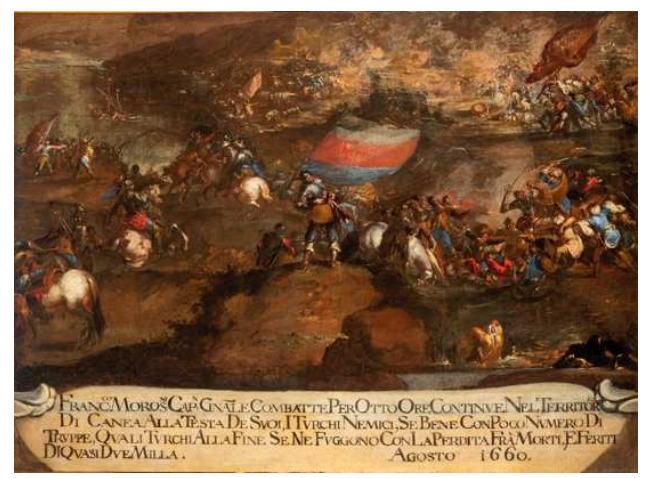

Fig. 1. Battaglia, Venezia, Palazzo Soranzo (Museo Correr, Cl. I, 1323).

\subsection{Storia e biografia in pittura}

Questo ciclo, insolito anche solo considerando il numero di tele che lo compongono, è certamente qualcosa di più di un insieme di pitture del cosiddetto genere delle battaglie. Il suo valore documentario è notevole sotto diversi punti di vista, il primo dei quali è strettamente storico.

Francesco Morosini, protagonista dei fatti rappresentati, fu senza dubbio una figura singolare della Venezia del XVII secolo (Gullino, 2012) legata a momenti di grande difficoltà e di grande entusiasmo popolare nella storia della Repubblica ${ }^{2}$. Morosini fu principalmente un uomo di guerra, per ben quattro volte capitano generale da mar, carica che si nomina nei periodi di guerra e che identifica il massimo comandante della flotta e delle operazioni militari navali e terrestri dell'esercito della Repubblica. In questa posizione, Morosini diresse le campagne militari durante la parte finale della guerra di Candia (Creta, durata dal 1645 al 1669), e quasi tutte quelle nel corso della guerra di Morea (Peloponneso, 1684-1693). Fu lo stesso Morosini a trattare nel 1669 la resa di Candia (Iraklion), la capitale del Regno, atto che pose fine al conflitto e determinò la perdita dell'isola che apparteneva a Venezia da 450 anni. Nella guerra di Morea, le conquiste territoriali di Morosini che portarono a
Venezia gran parte del Peloponneso (territori perduti già alla fine del Quattrocento) furono considerate da molti l'inizio di un possibile recupero dell' antico potere, un'idea che tuttavia aveva poco reale fondamento e che ebbe vita molto breve: la Morea fu infatti definitivamente persa solo pochi anni dopo, tra 1714-1718 (Infelise, Stouraiti, 2005; Pinzelli, 2016). Circostanza eccezionale, per la Repubblica, dal 1688 in poi il capitano generale da mar fu il doge in persona, la massima carica dello stato, benchè principalmente rappresentativa: l'elezione a doge raggiunse Morosini alla fine di aprile del 1688, mentre si trovava sul campo di battaglia in Morea. Francesco Morosini fu quindi ora pubblicamente messo sotto accusa, come avvenne per la conclusione della guerra di Candia, ora pubblicamente celebrato con il titolo di Peloponnesiaco e con il tributo di un busto, adhuc viventi, da collocare in palazzo Ducale.

Il ciclo delle tele Morosini si può dunque considerare una sorta di biografia per immagini poichè rappresenta sia gli episodi di vittoria che le sconfitte subite da Morosini e dal suo esercito nel corso delle due guerre.

\subsection{Registri figurativi}

Un secondo fattore di interesse riguarda i registri figurativi utilizzati nelle tele Morosini. Le pitture presentano immagini legate alla guerra ma di diversa natura: scontri navali (Milo, 1325; Nixia, 1357; Prevesa 1326; Candia 1322; luogo non precisato 1327 e 1333), scene di battaglia (Candia, 1320 e 1321; Megara, 1323; Canea, 1319; Schiato, 1330; fiume Aspro, 1335) e rappresentazioni territoriali e di paesaggio che si potrebbero meglio definire come 'ritratti' di fortezze (S. Maura, 1355; Prevesa, 1334; Chielefà, 1329; Calamata, 1337; Castel Tornese, 1356; Mistrà 1352; Bardugni, 1348; Egina 1354) e di città (Napoli di Romania, 1324; Navarino Nuovo, 1340; Modone, 1343).

Tutte queste immagini hanno evidenti rapporti con quelle diffuse nella pubblicistica di guerra, un fenomeno che si diffonde a Venezia e in tutta Europa con inedita ampiezza nel Seicento (Marasso, Stouraiti, 2001) ed è possibile individuare molte somiglianze tra le tele Morosini e le immagini stampate, per esempio, nelle opere di Coronelli, 
Albrizzi e Merian (Buratti 2019). In alcuni casi il percorso sembra inverso: non dalle stampe ai dipinti ma il contrario (ad esempio Bardugni, cfr. Buratti, 2019, pp. 318-319) anche se è indubbio che queste relazioni devono tenere conto delle fonti comuni a dipinti e incisioni costituite da un esorbitante numero di immagini manoscritte relative ai fatti di guerra: disegni di carattere ufficiale prodotti dall' apparato pubblico di governo oppure di origine privata e divulgativa, appartenenti a personalità e famiglie direttamente coinvolti nelle operazioni militari (Tonini, 2012; Molteni, 2019).

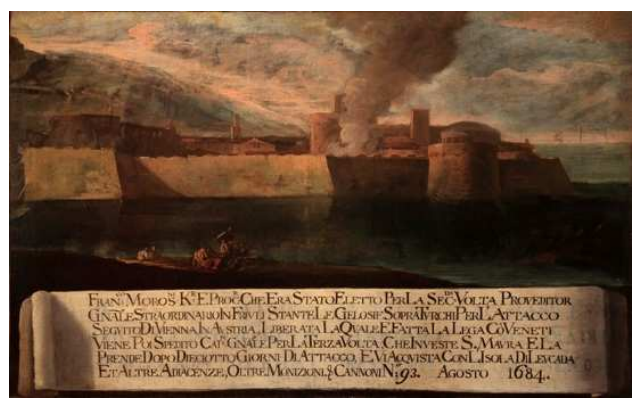

Fig. 2. Santa Maura, Venezia, Arsenale, Ammiragliato, (Museo Correr, Cl. I, 1355).

Un quarto tipo di immagini di guerra è costituito da scene di carattere ancor più strettamente tecnico-militare. Sono ben documentate nei dipinti del ciclo Morosini le particolari strategie nella dislocazione delle truppe e nelle strategie di attacco che Morosini impiegò, ad esempio trasferendo via mare e sbarcando le truppe a ridosso delle località da conquistare (Calamata, 1363; Gominizze, 1336; s.l. 1353; Patrasso, 1364) oppure attaccando le fortezze marittime contemporaneamente da terra e con il cannoneggiamento navale (Volo, 1358; Torron, 1360; Cisme (Çeşme), 1328; Castel Ruggio, 1359; Calami, 1332; Calogero, 1361; Santa Veneranda, 1362).

Ancora più specialistiche sono tre tele basate direttamente su disegni eseguiti da ingegneri e tecnici militari, fogli che riportano con dovizia di particolari la topografia dei luoghi, le posizioni degli eserciti avversari, le fasi dell'attacco, la disposizione delle opere di fortificazione permanenti e provvisionali. Questi disegni documentano allo stesso tempo l'attività degli ingegneri militari (progetti e opere realizzate sotto il loro controllo); le loro composite competenze professionali in ambito militare e in architettura (in particolare la loro abilità nel disegno, in alcuni casi di alto livello); infine manifestano le relazioni che gli ingegneri intrattengono sia con $\mathrm{i}$ «capi da guerra» che con gli esponenti politici dell' apparato militare della Repubblica, ai quali i fogli sono spesso indirizzati.

Probabilmente la scelta dei soggetti di queste pitture non è casuale: Candia (inv. 1365) è il più importante assedio del secolo, divenuto leggendario per la sua durata, mentre Corone e Negroponte, sono due episodi connotati da esiti e ripercussioni opposti che ben descrivono anche le alterne fortune della carriera militare di Morosini. La conquista di di Prevesa e Santa Maura, avamposti che attraverso l'isola di Leucada aprono al golfo di Lepanto e al Peloponneso, la conquista di Corone nel 1685 , aprì una serie di fortunate azioni contro le città di Morea nelle campagne di guerra degli anni 1686 e 1687 e fu ampiamente celebrato in Venezia. Al contrario, il fallimento dell'assedio di Negroponte nell'estate del 1688, segna l'avvio della parabola discendente della guerra che proseguirà ancora per qualche tempo ma senza grandi imprese.

\section{La conquista di Corone (1685)}

La presenza nel ciclo morosiniano di due dipinti dedicati a Corone è probabilmente legata al fatto che gli episodi decisivi delle sorti dell'assedio ebbero due scenari diversi. A Corone infatti l'esercito veneziano attaccò e si difese su due fronti: contro l'accampamento ottomano posto "nel luogo nominato Dervis Mehmet", due miglia alle spalle di quello veneziano (Fig. 3) e contro la fortezza situata sull'estremità del promontorio e ben difesa: l'esplosione del torrione centrale del fronte di terra della fortezza (MC, Cl. I, 1338) è l'episodio che conclude l'assedio con la resa della città (Locatelli, 1691, pp. 124-158; Garzoni, 1705, pp. 99-111; Coronelli, 1687, pp. 18-27; Pinzelli, 2016, pp. 171-184).

Quasi certamente gli ottomani non ebbero il tempo di preparare a Corone una difesa efficace poichè la campagna del 1685 si era aperta con l'intenzione di attaccare Modone. Tuttavia, riconosciuta questa piazza dal generale da sbarco Claude de Saint Paul Longueville (già veterano della 
guerra di Candia) e dall'ingegnere Milhau Verneda, sembra che la mancanza di un luogo adeguato allo sbarco, abbia suggerito, il 22 giugno (Locatelli, 1691, p. 124) di deviare l'attacco su Corone, dove l'armata giunge solo due giorni dopo. Dopo lo sbarco di oltre settemila uomini, l'accampamento veneziano si posiziona tra la fortezza e l'abitato di Corone: il borgo affacciato sul mare, separato dalla fortezza e non fortificato, come spesso accade nei territori di Levante. Il controllo del borgo, occupato con una certa facilità, manteneva aperte anche le comunicazioni marittime.

La formazione di un campo ottomano è quindi il risultato della riorganizzazione difensiva di $\mathrm{Co}-$ rone. Prende la posizione un'avanguardia ottomana di 1200 soldati, guidata da Mustafa Bassà, ma si raccolgono rinforzi nel territorio, comandando che ogni famiglia debba inviare un uomo armato "provveduto di badile, zappone e sacco da esser impiegati nel tirar cannone e alzar terreno" contro il campo veneziano (Locatelli, 1691, p. 135); infine si raccolgono truppe a Patrasso e presso le fortezze più prossime di Zarnata e Chielefà che giungono con Calil, Seraschier di Lepanto. I veneziani sono quindi costretti a rinforzare le difese del proprio accampamento verso la campagna e a sostenere diversi scontri su quel fronte, mentre continuano i lavori intrapresi contro l'obiettivo principale, le mura della fortezza.

In queste operazioni sono determinanti le opere funzionali agli attacchi costruite sotto la direzione degli ingegneri militari con l'impiego di strumenti e materiali propri delle opere provvisionali. Si dispongono sul terreno verso l'accampamento ottomano linee di trincea, bonetti e batterie di artiglieria, mentre verso la città attraverso gallerie protette da strutture in legno, gabbioni, sacchi di terra si procede alla costruzione dei fornelli di mina. Il 23 luglio si decide di far volare la mina costruita nei pressi di quella che sembra l'unica porta di accesso alla fortezza, nelle mura marittime verso il borgo ("a fronte dell'ingresso per mezo il Mezit", Locatelli 1691, p. 140) che però non ottiene i risultati attesi "non appianò la strada necessaria alla salita".

Nei giorni seguenti Milhau Verneda lavora ancora sul luogo di questa breccia (costruisce un'altra "galleria di mantiletti" ma viene ferito e l'opera resta priva della sua assistenza) mentre l'ingegner Giovanni Bassignani dirige la costruzione di un'altra mina "sotto il gran scarpone".

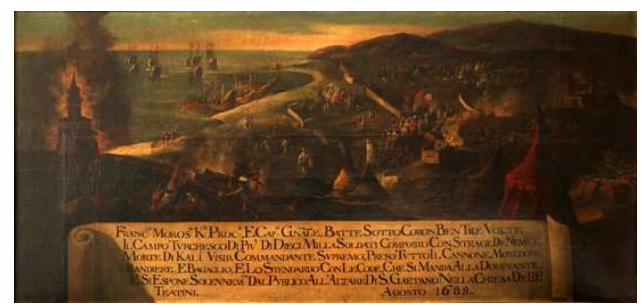

Fig. 3. Conquista del campo ottomano di Corone (Venezia, Museo Correr, Cl. I, 1331).

La maggior parte degli scontri sembra però sull'altro fronte, lungo la linea di circonvallazione che attraversa la penisola e delimita il campo veneziano. La conquista del campo ottomano il 7 agosto, con un'azione notturna e uno stratagemma porta «un opulente bottino, quantità di bandiere, il principale stendardo, code di comando, sontuosi padiglioni, armi, cavalli, somma di denaro» (Locatelli, 1691, p. 149). Il giorno successivo si decide di far brillare la mina di Bassignani sotto allo scarpone che "con orribile scotimento, spalancata una grande breccia, [aprì] via capace da introdurre le milizie dentro la piazza".

Il campo ottomano rappresentato nella tela Morosini (Fig. 3) è un accampamento fortificato molto simile a quello veneziano, il che è molto plausibile. La raffigurazione è molto precisa anche nella descrizione dell'appoggio della flotta sottile (che ebbe un ruolo diversivo) e del bonetto elevato che fu il teatro di un decisivo scontro tra i due eserciti poco prima dell'assalto e dell' abbandono del proprio campo da parte dell'esercito ottomano.

La seconda immagine ritrae invece da terra lo scoppio spettacolare del torrione. Il pittore avrebbe potuto basarsi su molte immagini di Corone diffuse in questi anni, per esempio nelle opere di Coronelli e di Tibaldi, ma nessuna impiega questo punto di vista nè ritrae in modo simile queste strutture.

Le due tele, pur impiegando un linguaggio non tecnico ma narrativo, usano invece sicuramente come modello la mappa dell' assedio di Corone di- 
segnata da un testimone dei fatti, Giovanni Bassignani e dedicata a Lorenzo Venier che con Giorgio Benzoni era stato uno dei due provveditori in campo a Corone (Fig. 4). Non solo il disegno presenta fasi e opere realizzate durante l'assedio ma nei due riquadri in basso compaiono il "gran torrione" in forme del tutto simili a quelle del dipinto e una veduta del fronte di terra della città dopo l'esplosione, l'unica esistente, per quanto finora noto.

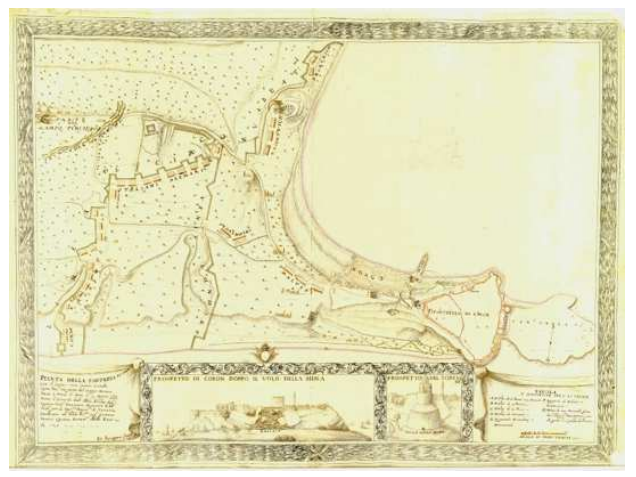

Fig. 4. Giovanni Bassignani, Assedio di Corone (in Francesco Morosini 2019).

\section{Il fallimento di Negroponte (1688)}

Per assicurare il controllo dei territori dell'entroterra conquistati nel corso della guerra di Morea, Morosini considera luoghi strategiamente essenziali due città, oltre a quelle portuali sulle coste del Peloponneso: Corinto, conquistata nell'agosto del 1687, e Negroponte "quella piazza che tirava seco un regno" (Locatelli, 1691, p. 345). Dopo la conquista di Corinto, nell'agosto del 1687, Morosini cerca subito di dirigere l'armata verso Negroponte ma la consulta di guerra (composta dai più alti gradi di comando dell'esercito) si oppone, considerando la città troppo ben difesa e la stagione troppo avanzata (Pinzelli, 2016, pp. 152-153). Solo dopo il successo della campagna di Atene, e una volta eletto doge Morosini all'unanimità il 3 aprile 1688, la consulta del 27 giugno 1688 decise di attaccare la principale piazzaforte dell'isola di Eubea, Negroponte (Khalkis).

L'assedio di Negroponte è particolarmente interessante per l'assetto delle difese approntato dal governo ottomano che sfrutta con abilità un contesto territoriale complesso, marittimo e terrestre. La città è situata sull'isola che in quel punto è così vicina all'entroterra che un ponte (fortificato) la collega all' antica Beozia.

Il territorio collinare alle spalle della città, insieme ad aree paludose, costringe $i$ veneziani a un assedio particolarmente difficile ed elaborato, condotto oltretutto in condizioni climatiche difficili per il caldo della stagione estiva. Truppe, capi militari e ingegneri sono decimati dal contagio malarico oltre che dai combattimenti. Il primo luglio 1688 l'armata comincia il blocco dei due canali che conducevano a Tebe e verso nord a Volo, si stabilisce il primo accampamento, a una buona distanza dalla città e dal borgo, situato all'esterno alla cinta fortificata, sul fianco nord della penisola. Cominciano i preparativi al conflitto che si protrae per tutta l'estate e oltre il periodo consueto delle campagne di guerra, fino al 20 ottobre quando l'armata, dopo l'ennesimo tentativo fallito, leva l'assedio.

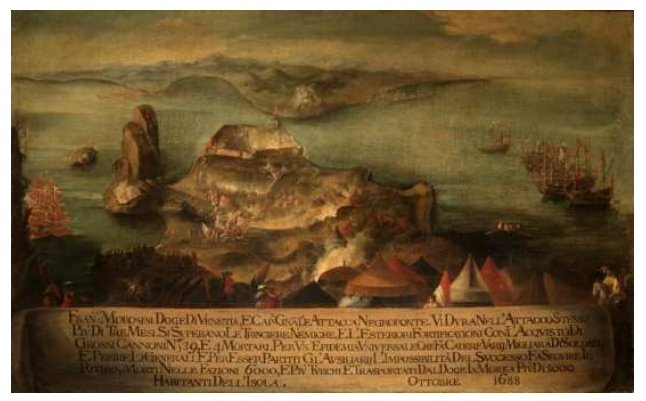

Fig. 5 Assedio di Negroponte, Venezia, Circolo Ufficiali della Marina (Museo Correr, Cl. I, 1351).

\subsection{Le difese ottomane}

Certamente anche gli ottomani consideravano $\mathrm{Ne}$ groponte un luogo strategicamente importante, e la città era quindi perfettamente consapevole di un possibile attacco. Secondo le fonti, l'assetto delle difese di Negroponte fu organizzato da Girolamo Galoppi di Guastalla, disertore e rinnegato, la cui attività come ingegnere proseguirò nell'esercito ottomano anche dopo Negroponte (Pinzelli, 2016, pp. 153, 183) ma non è tuttavia documentata presso Venezia. A lui spetterebbe la costruzione 
del forte Carababà, situato sulla terraferma a dominare la città e i due canali. Il forte sicuramente non esisteva nella ricognizione del territorio di Negroponte conservata nelle carte del generale Johann Rudolf Werdmüller (1614-1677) probabilmente redatta molti anni prima. $\mathrm{Ci}$ offre la sua pianta l'ingegnere Milhau Verneda (BNM, It. VII 94 10051, n. 109). La fortezza fu eseguita su impianto irregolare -la fusione di un quadrato e un triangolo- con torri di diversa pianta agli angoli e un torrione principale esagonale. Lo stesso ingegnere, in un disegno complementare, dimostra come si dovrebbe attaccare il forte (BNM, It. VII 94 10051, n. 108). Nulla fu eseguito per la sospensione dell'attacco e, in ogni caso, il ruolo del Carababà fu piuttosto marginale nello sviluppo delle operazioni di assedio.

Dunque, tornando alla città, erano state preparate diverse infrastrutture per la sua difesa, soprattutto difese esteriori alle mura dalla parte verso il borgo. Si era costruita una linea di trincea palificata che attraversava la penisola, con quattro batterie e almeno tre mine. Inoltre un'altra batteria era stata collocata, su una collina prossima alla città verso il canale di Tebe, dalla quale si sarebbe potuto battere la città. In vicinanza delle mura cittadine gli ototmani avevano costruito una controscarpa con palificata che a sinistra, verso la collina, era completata da tre rivellini. Tutto questo è conquistato dall'esercito alleato il 13 luglio, grazie alle informazioni avute da tre fuggitivi greci secondo i quali "in buona parte [avevano] terrapienate le mura, ed alcune torri, con cannone da per tutto. Che havessero apparecchiate mine sotto l'Appanau, ò sia porta" e che ai "lavori esterni già perfezzionati n'aggiungevano altri di nuovo"'.

\subsection{I tempi dell'assedio}

I resoconti dell'assedio narrano molti episodi relativi alla conquista di luoghi e postazioni rilevanti poi perdute e di nuovo riprese. I bombardamenti veneziani iniziarono il 30 luglio. Nei giorni successivi l'attacco si incentrò sulla prima linea di difesa fatta dai turchi e sul tentativo di conquistare lo scoglio in bocca al porto, il che accadrà il 7 agosto. L'avanzamento veneziano segnò un passo importante il 16 agosto quando fu presa la prima linea di trincea turca, vicina ai mulini a vento: gli ottomani, considerandola troppo importante, lanciarono un'offensiva il giorno seguente, riconquistandola. Per questa ragione, lo stesso giorno (17 agosto) si tenne una consulta in cui Morosini, senza l'approvazione unanime di tutti i capi da guerra, decise di eseguire il 20 agosto un attacco generale alle trincee.

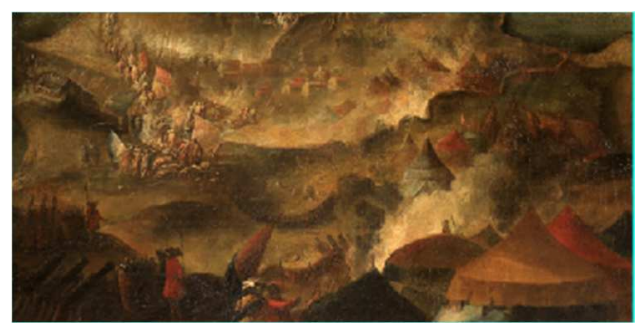

Fig. 6 Assedio di Negroponte, dettaglio.

L'obiettivo successivo dei veneziani fu arrivare alla fossa. I lavori di avvicinamento si iniziarono il 27 agosto e nei giorni seguenti. In particolare, il 5 settembre si registrò un attacco di 500 turchi verso il borgo, respinti dai veneti e inseguiti fino alla fossa. Nei giorni successivi il bombardamento si concentrò sul torrione da mar sinistro, aprendo un breccia che permise ai veneziani di salirvi l'8 settembre. Questo successo non potè essere mantenuto perché il sito era scoperto, di dimensioni ridotte ed erano "custodite e difese le porte che comunicavano sopra le mura, onde furono astretti di retrocedere" (Locatelli, 1691, p. 123). Il 10 settembre, infine, le gallerie iniziate nella controscarpa sboccarono nel fossato, momento in cui si iniziò il bombardamento del torrione destro e della sua cortina per mezzo di due cannoni "al bordo del fosso" e si iniziò la galleria di mina. Ma a questo punto l'assedio soffrì la mancanza di ingegneri capaci di dirigere i lavori (in maggior parte morti sul campo), la morte di vari generali, tra i quali Königsmarck (15 settembre), e la partenza di alcune truppe ausiliarie. Nonostante tutto, il 12 ottobre si terrà un attacco generale nella fossa per tentare gli assalti alle brecce, ma tutti i tentativi furono respinti dagli ottomani poichè gli attacchi veneziani erano troppo vicini alle traverse e falsebraghe nemiche. Il fallimento di questa operazione, i danni causati dai cannoni turchi alle navi che attaccavano nello stretto e l'impossibilità di riorganizzare l'esercito per mancanza di persone 
che rivestissero adeguatamente la cariche necessarie, causeranno la sospensione dell'attacco (Locatelli, pp. 140-143). Pochi giorni dopo si ritirarono i cannoni vicini alla controscarpa e si imbarcarono truppe e civili in fuga per passare a Napoli di Romania.

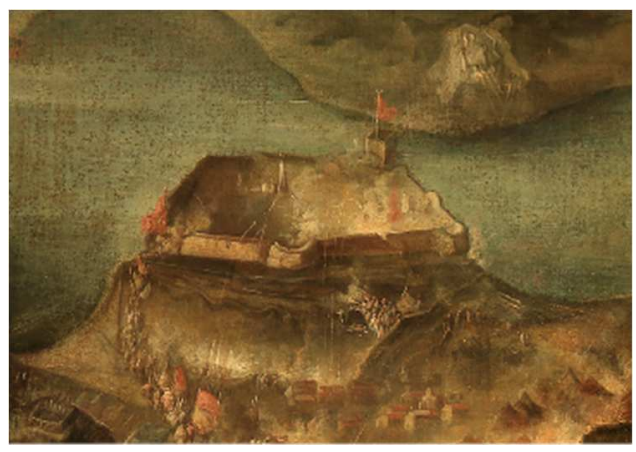

Fig. 7 Assedio di Negroponte, dettaglio.

\subsection{La tela del ciclo Morosini e la cartografia di guerra}

All'interno del ciclo pittorico morosiniano, la tela dedicata a Negroponte (Figg. 5-7) illustra i principali eventi dell'assedio così come sono trasmessi dalla cartografia di guerra conservata alla Biblioteca Nazionale Marciana e all'Archivio di Stato di Venezia. La tela colloca a lo spettatore a est, da dove si apre il panorama verso ovest su tutto il territorio, il borgo, la città. In primo piano si osserva il primo campo veneziano (Biblioteca Nazionale Marciana, Venezia, It. VII 94 10051, n. 104 "primo accampamento de schiavoni / primo accampamento di quattro battaglioni" e n. 110 "M. Primo campamento veneto tra la colina del monte et ne giardini vicino al paludo"), che era situato tra una collina, la zona chiamata "Giardini”, e un' area paludosa vicino al mare, occupando così tutto il tratto più ristretto della penisola. La posizione del campo, anche se lontano dalla città, non è casuale, dal momento che quella zona consentiva dominare la collina con il posizionamento di una batteria (B.N.M. It. VII 94 10051, n. 110 “N. Batterie venete sopra il monte che domina le turchesche ma troppo lontane"), di appropriarsi di diverse fonti di acqua dolce e di bloccare il passaggio di alcune strade principali che conducono direttamente alla città per evitare l'arrivo di rinforzi e soccorsi.
Il lavoro di approccio alla città iniziò il 5 agosto (Locatelli, p. 106). Dalla prima linea di contravalazione (BNM, It. VII 94 10051, n. 110, "L") tra il 12-13 agosto si iniziarono a scavare le prime trincee in direzione alla palificata turca che proteggeva il borgo (n. 110, "A"; Locatelli, 1691, p. 108). Questa linea esterna è ben disegnata sulla tela, in cui si vede che un gruppo di truppe veneziane l'ha superata e sta andando verso la torre di sinistra, sulla quale è stata messa una bandiera che sembra essere quella del leone di san Marco. La tela riporta quindi contemporaneamente due episodi che ebbero luogo in momenti diversi: quello del 20 agosto, quando si diede l'attacco generale sulla trincea (evento protagonista anche di un disegno conservato presso la Bibliothèque Nationale de France P179656, Vd-5 (3)-Fol. https://gallica.bnf.fr/ark:/12148/btv1b6903920n) e quello dell'8 settembre, quando l'attacco guidato dall'ingegnere Pietro Romagnato riesce a rilevare per qualche ora il torrione da mar sinistro. La tela mostra anche la linea di trincea realizzata dall'esercito veneziano sulla destra, tra il borgo e la città, dove si vede un gruppo di soldati: questo episodio sembra riferirsi a quanto avvenne il 5 settembre dalla parte del borgo quando i turchi, superata la prima trincea, vennero respinti dai veneti.

Per quanto riguarda la città di Negroponte, i diversi disegni raccolti la mostrano, come riportato dalle cronache, "fasciata di muraglie ad uso de' tempi antichi, con molte torri all'intorno, che la rendono forte, e resistente" (Diario dell'assedio, 1688, p. 27). Questa descrizione è ricorrente nei disegni conservati alla Biblioteca Marciana (BNM, It. VII 94 10051, n. 111 e 113) ma non trova un corrispettivo esatto nella tela. Invece, si può osservare come la batteria veneziana abbia provocato due brecce nelle torri delle estremità settentrionali e verso la terraferma meridionale, indicata nel disegno conservato nell' archivio Grimani ai Servi presso l'Archivio di Stato di Venezia (Archivio di Stato, Venezia, Arch. Grimani ai Servi, f. 57/172, fasc. D/d, pos. 59, segnato con il n. 11). Nella parte più esteriore si trovava anche una fossa bagnata direttamente dal mare, a sua volta protetta da una palificata e controscarpa. 


\section{Note}

Lo studio è opera comune. La scrittura dei paragrafi 1 e 2 è di E. Molteni, il paragrafo 3 di A. Pérez Negrete.

${ }^{1}$ Ventitré tele sono state esposte alla mostra Morosini in guerra a Candia e in Morea, Venezia, $\mathrm{Pa}$ lazzo Corner Mocenigo - Guardia di Finanza, Comando Regionale Veneto, 12 luglio-5 novembre 2019.
2 (2019). Francesco Morosini 1619-1694. L'uomo, il doge, il condottiero, Poligrafico e Zecca dello Stato Italiano Ed., Roma.

3 (1688). "Diario dell' assedio della Città di Negroponte sotto la Condotta del Serenissimo Francesco Morosini Prencipe di Venezia", Ragguaglio giornaliero delle trionfatrici, \& inuitissime armate venete maritime, \& terrestri co' suoi acquisti a pontino distinti contro la potenza ottomana, Venezia, p. 23.

\section{Bibliography}

Arisi, F. (1982). "Il punto su F. M. detto il Brescianino delle Battaglie”, in Memorie bresciane, II, 1, pp. 140-156.

Buratti, B. (2019). "Le campagne militari nel ciclo pittorico delle collezioni Morosini”, in Francesco Morosini, pp. 229-329.

Coronelli, V. (1687). Memorie Istoriogeografiche della Morea, Venezia.

Garzoni, P. (1705). Istoria della Repubblica di Venezia in tempo di Sacra Lega, tom. I.

Gullino, G. (2012). "Voce Morosini Francesco", in Dizionario Biografico degli Italiani, Istituto Italiano Enciclopedia Treccani Ed., Roma, vol. LXXVII.

Infelise, M.; Stouraiti, A., eds. (2005). Venezia e la guerra di Morea. Guerra, politica e cultura alla fine del '600, F. Angeli Milano Ed.

Locatelli, A. (1691). Racconto istorico della veneta guerra in Levante diretta dal valore del serenissimo principe Francesco Morosini capitan generale la terza volta per la Serenissima Repubblica di Venetia, Colonia a spese di Girolamo Albrizzi.

Marasso, L.; Stouraiti, A., eds. (2001). Immagini dal mito: la conquista veneziana della Morea, 1684-1699, catalogo della mostra (Venezia, Querini stampalia), Fondazione scientifica Querini stampalia Venezia Ed., Venezia.

Molteni, E. (2019). "Le tele Morosini e le immagini della guerra di Morea: una testimonianza dei rapporti tra esercito e società nel XVII secolo", in Francesco Morosini, pp. 364-375.

Pignatti, T. ed. (1960). Il Museo Correr di Venezia. Dipinti del XVII e XVIII secolo, Istituto di Storia dell'Arte Fondazione Giorgio Cini Venezia Ed.

Pinzelli, E. (2016). Venise et la Morée: du triomphe à la desillusion (1684-1718), PhD, Université Aix-Marseille I, pp. 97-109.

Tonini, C. (2012). La cartografia storica dagli archivi familiari alle raccolte del Museo Correr di Venezia, in Agazzi, M.; Frank, M.; Marinelli, S., eds., Archivi di disegni disegni in archivio, Il Prato, Saonara, pp. 60-72.

Tonini, C. (2013). "Palazzo Morosini: la difficile eredità di Loredana Morosini Gatterburgh", Bollettino dei Musei Civici Veneziani, 3 ser. 8, pp. 102-106. 